\title{
Unfolding the band structure of GaAsBi
}

R. Maspero, S. J. Sweeney and Marian Florescu

Advanced Technology Institute and Department of Physics, University of Surrey, Guildford, Surrey, GU2 7XH, United Kingdom

E-mail: m.florescu@surrey.ac.uk

15 July 2016

\begin{abstract}
Typical supercell approaches used to investigate the electronic properties of $\operatorname{GaAs}_{(1-x)} \mathrm{Bi}_{(x)}$ produce highly accurate, but folded, band structures. Using a highly optimized algorithm, we unfold the band structure to an approximate $\mathrm{E}(\mathbf{k})$ relation associated with an effective Brillouin zone. The dispersion relations we generate correlate strongly with experimental results, confirming that a regime of band gap energy greater than the spin-orbit-splitting energy is reached at around $10 \%$ bismuth fraction. We also demonstrate the effectiveness of the unfolding algorithm throughout the Brillouin zone (BZ), which is key to enabling transition rate calculations, such as Auger recombination rates. Finally, we show the effect of disorder on the effective masses and identify approximate values for the effective mass of the conduction band and valence bands for bismuth concentrations from $0-12 \%$.
\end{abstract}

Submitted to: J. Phys.: Condens. Matter 


\section{Introduction}

The rising call for greener technology combined with the ever growing demand for a faster, more widespread Internet has put pressure on the telecommunications industry to improve the efficiency of its devices. One area capable of dramatic improvement is in the active materials of laser and detection equipment, where it has been shown that lasers, at a wavelength of $1.55 \mu \mathrm{m}$, lose as much as $80 \%$ of the current required to lase to Auger recombination[1]. It is believed that the dominant contribution to this loss mechanism (CHSH Auger recombination[2]) can be suppressed by increasing the spin-orbit-splitting energy, $\Delta_{s o}$, to greater than the band gap energy, $\mathrm{E}_{g}[3]$.

Auger recombination is a non-radiative process in which a conduction band electron recombines with a valence band hole and transfers the subsequent recombination energy to a third carrier, which is consequently excited higher up in its respective band. The type of Auger recombination dominant in $\operatorname{InGa}(\mathrm{Al}) \mathrm{As}(\mathrm{p}) / \mathrm{InP}$ based telecommunication devices $(\mathrm{CHSH})$ is conveyed in Fig. 1.

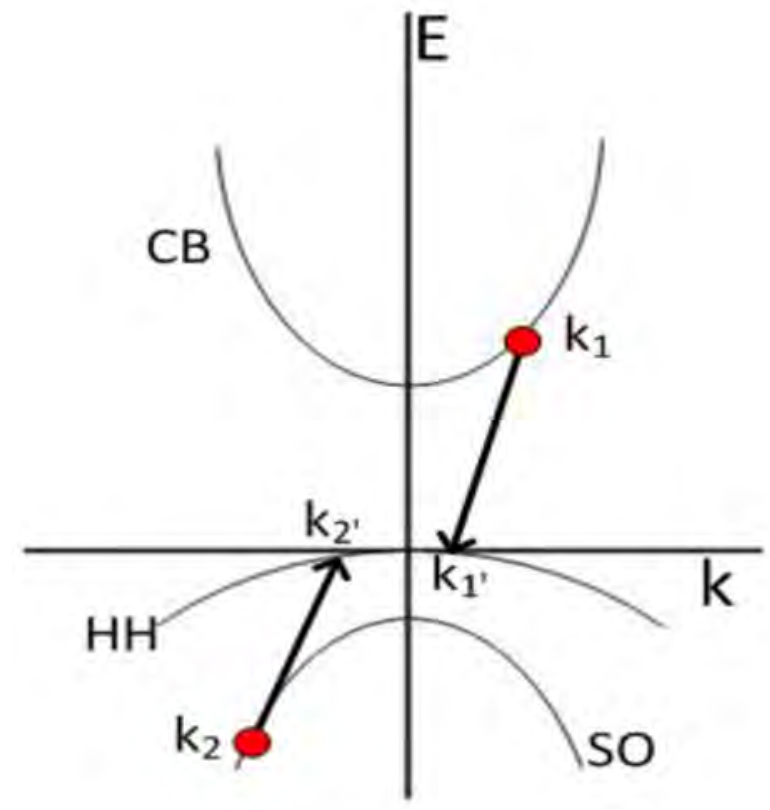

Figure 1. (Color online) CHSH Auger recombination occurs when an electron from the conduction band $\left(k_{1}\right)$ recombines with a hole from the heavy-hole band $\left(k_{1}^{\prime}\right)$, with a subsequent excitation of a hole from the split-off band $\left(k_{2}\right)$ to an excited state in the heavy-hole band $\left(k_{2}^{\prime}\right)$. This effect can be suppressed when the SO band has been lowered to an extent that the $k_{2}$ electron can no longer transition to the $\mathrm{HH}$ band, while conserving the energy and momentum from the $k_{1}$ to $k_{1}^{\prime}$ transition.

$\operatorname{GaAs}_{(1-x)} \mathrm{Bi}_{(x)}$ alloys have been proposed as a solution for reducing the CHSH Auger recombination, which can be dramatically diminished when $E_{g}<$
$\Delta_{S O}[4,5] . \quad$ GaAsBi shows a massive decrease in $\mathrm{E}_{g}$ of $60-90 \mathrm{meV} / \% \operatorname{Bi}[6,7,8]$ and an increase of $30-60$ $\mathrm{meV} / \% \mathrm{Bi}$ in $\Delta_{s o}[9]$. The movement of bands has been described by band anti-crossing (BAC), where an anticrossing interaction occurs between a bismuth defect level and the host valence band. This rapid translation of the bands allows $\Delta_{s o}>\mathrm{E}_{g}$ at around $10 \%$ bismuth fraction $[9,10]$. Furthermore, the material emits in the telecommunications wavelength region $(1.55 \mu \mathrm{m})$ at this percentage bismuth[10].

Theoretical studies on this material have shown how BAC can explain the movement of the bands $[9$, $11,12,15]$ and finer detail into the response of the electronic structure on defects, like bismuth clustering[9], that correlates well with experimental results. These studies have used a variety of band structure models including LDA $+\mathrm{C}[12]$, pseudopotential[16], k·p $\mathbf{p}[15]$ and, as in this work, tightbinding[9]. In order to model systems with defects or general inhomogeneities, a supercell approach is often adopted. This is a construction of stacked primitive cells, that are generally non-identical, along the lattice vectors, which is employed to simulate defects and other inhomogeneities. The supercell approach is used to model ternary alloys in order to account for the different configurations of the three constituent atoms. Alternative methods are available, like the virtual crystal approximation (VCA) but generally fail to reproduce the movement of the bands as recorded in experiments, for alloys where the dopant has a strong perturbation effect. However, the supercell approach also has a disadvantage, which comes in the form of zone folding[17, 18, 19].

Zone folding occurs because of a shrinking of the BZ of supercells. Larger real-space dimensions lead to a smaller reciprocal space causing information to be 'folded' in from lower order BZs[19]. Folding of a supercell band structure makes extraction of information near impossible as the $\mathrm{E}(\mathbf{k})$ relationship is lost and the dispersion becomes a dense block of bands (Fig. 2). Moreover, many other physical quantities/phenomena, such as gain and carrier mobility, require the knowledge of the true $\mathrm{E}(\mathbf{k})$ relationship making it essential be able to unfold the supercell band structure. In this work, we employ the formalism developed in Refs. $[17,18,19]$ to unfold the band structure of $\operatorname{GaAs}_{(1-x)} \mathrm{Bi}_{(x)}$ in order to demonstrate the potential to extract important material properties.

\section{Methodology}

To generate the band structure of $\mathrm{GaAs}_{(1-x)} \mathrm{Bi}_{(x)}$, a supercell consisting of a large number of distinct primitive cells is built. Each primitive cell contains 
a $\mathrm{Ga}$ atom and either an $\mathrm{As}$ or $\mathrm{Bi}$ atom, which is chosen on a weighted coin-toss basis defined by the required bismuth fraction. The supercell needs to be sufficiently large that all possible configurations of the three atoms are reasonably represented and the effect of symmetry imposed by the supercell is negligible. The structure is then relaxed through minimisation of the complete lattice energy using the valence force field (VFF) model[20]. The list of parameters used for the relaxation are shown in Table 1 .

Table 1. Scaling and VFF parameters for the $s p^{3} s^{*}$ tightbinding Hamiltonian. Subscripts $a$ and $c$ represent the anion and cation, respectively. $s, p$ and $s^{*}$ denote the respective orbitals, and $\sigma$ and $\pi$ are parallel and perpendicular components of that parameter, respectively $[9,21]$.

\begin{tabular}{lll}
\hline Name & GaAs & GaBi \\
\hline Scaling Parameters & & \\
\hline$\eta_{s s \sigma}$ & 3.512 & 3.66 \\
$\eta_{s p \sigma}$ & 4.3 & 4.08 \\
$\eta_{p p \sigma}$ & 3.2042 & 2.2 \\
$\eta_{p p \pi}$ & 4.2357 & 3.24 \\
$\eta_{s^{*} \sigma}$ & 5.5 & 5.5 \\
\hline VFF Parameters & & \\
\hline$\alpha_{0}\left(\mathrm{~N} \mathrm{~m}^{-1}\right)$ & 41.49 & 34.0 \\
$\beta_{0}\left(\mathrm{~N} \mathrm{~m}^{-1}\right)$ & 8.94 & 5.0 \\
$A$ & 7.2 & 8.2 \\
$B$ & 7.62 & 7.62 \\
$C$ & 6.4 & 6.4 \\
\hline
\end{tabular}

Using this structure, a tight-binding Hamiltonian is then defined and diagonalised to find the dispersion relation. The Hamiltonian evaluates the $s p^{3} s^{*}$ orbitals, including the spin-orbit interaction, and accounts for the varying inter-atomic distances but only for nearest neighbours. This is achieved by varying the interaction parameters by $\left(d_{0} / d\right)^{\eta}$, where $d$ is the inter-atomic bond length, $d_{0}$ is the unstrained bond length and $\eta$ is a scaling parameter dependent on the orbital interaction being considered. This relaxation procedure is highly relevant for $\operatorname{GaAs}_{(1-x)} \mathrm{Bi}_{(x)}$ alloys since the $\mathrm{Bi}$ atoms are much larger than As atoms and therefore induce a large amount of strain in the crystal. All the parameters used for the tight-binding Hamiltonian are listed in Table 1 and Table 2. The parameters from Refs. [9, 21] were optimized to reproduce the energy level shift shown by experiment[10] and the effective masses in the original binary compounds.

All tight-binding and strain relaxation calculations were performed on an adapted fifth edition of the NanoElecronics Modeling tools (NEMO5)[21], which specializes in atomistic calculations of the electronic and structural properties of systems on the nanoscale. The Elemental library[22] was incorporated into NEMO5 to perform a highly-efficient parallel diagonal-
Table 2. Lattice constants, self energies and interaction energies for the $s p^{3} s^{*}$ tight-binding Hamiltonian. Subscripts $a$ and $c$ represent the anion and cation, respectively. $s, p$ and $s^{*}$ denote the respective orbitals $[9,21]$.

\begin{tabular}{lll}
\hline Name & $\mathrm{GaAs}$ & $\mathrm{GaBi}$ \\
\hline Lattice Constant $(\AA)$ & 5.65325 & 6.328 \\
\hline Self-Energies $(\mathrm{eV})$ & & \\
\hline$E_{s, a}$ & -8.6336 & -8.3774 \\
$E_{s, c}$ & -2.9474 & -5.6126 \\
$E_{p, a}$ & 0.9252 & -0.1256 \\
$E_{p, c}$ & 3.5532 & 1.694 \\
$E_{s^{*}, a}$ & 7.0914 & 6.1262 \\
$E_{s^{*}, c}$ & 6.2 & 5.8164 \\
\hline Interaction Energies $(\mathrm{eV})$ & & \\
\hline$s s \sigma$ & -1.6835 & -1.3425 \\
$p p \sigma$ & 2.95 & 2.0003 \\
$p p \pi$ & -0.7420 & -0.6354 \\
$s_{a} p_{c} \sigma$ & 2.3920 & 2.3567 \\
$s_{c} p_{a} \sigma$ & 2.4200 & 1.2025 \\
$s_{a}^{*} p_{c} \sigma$ & 2.0400 & 1.8051 \\
$s_{c}^{*} p_{a} \sigma$ & 1.7700 & 0.5100 \\
$\lambda_{a}$ & 0.405 & 2.0142 \\
$\lambda_{c}$ & 0.165 & 0.1152 \\
\hline
\end{tabular}

isation of the Hamiltonian and an unfolding algorithm was developed and integrated to unfold the band structure as it was calculated.

The supercell tight-binding calculation generates a folded band structure, where this loss of a continuous $\mathrm{E}(\mathbf{k})$ relation complicates most forms of analysis. It becomes apparent that unfolding the band structure is essential to theoretically investigate the properties of $\operatorname{GaAs}_{(1-x)} \mathrm{Bi}_{(x)}$ and other ternary alloys. A possible method of unfolding the band structure is through a brute force approach by checking continuity of bands at folding and band-crossing points. This can be achieved by analysing the orthogonality of neighbouring eigenvectors, where eigenvectors of the same band will have a high eigenvector overlap. However, this method does not unfold to an intuitive $3 \mathrm{D}$ wavevector (as it is unfolded in 1D) and also does not account for the broadening of bands caused by the disorder. This method also becomes more complex with larger supercells as the increasing density of bands requires more checks and a smaller tolerance for the overlap. Therefore, an alternative method is applied that successfully unfolds any size perfect supercell (all constituent primitive cells are identical) in all directions [17, 18, 19], as shown in Fig. 2 for a 1458 atom GaAs supercell.

The same formalism can be applied to both artificially constructed supercells and imperfect supercells associated with disordered alloys. In the case of disordered systems, the resultant band structure can 

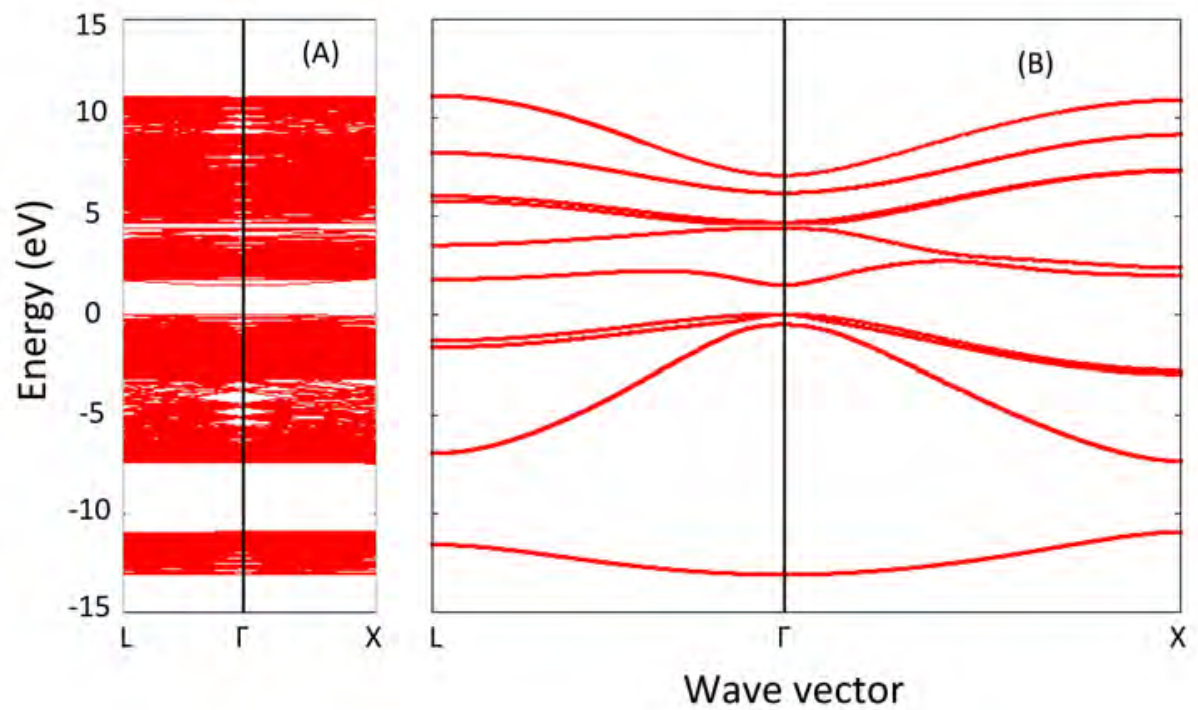

Figure 2. (Color online) The band structure of a 1458 atom perfect GaAs supercell calculated using $s p^{3} s^{*}$ orbitals. (A) Folded band structure with very few identifiable features (B) Unfolded band structure using the algorithm developed here, which reproduces the results obtained from a single primitive cell calculation.

no longer be exactly unfolded because a primitive-cell band structure imposes a level of symmetry that does not exist in a supercell. In other words, the wavevectors, $\mathbf{k}$, of a standard band diagram are no longer good quantum numbers in the supercell case. Nonetheless, an effective band structure (EBS) can be generated, which gives a distribution for each of the energy levels representing the statistically probable relation between the energy and momentum for electrons[18].

In the following, we present a brief description of the unfolding methodology. For simplicity, we consider here a bulk crystal with a primitive cell described by the lattice vectors $\mathbf{a}_{i}$ and the reciprocal lattice vectors $\mathbf{b}_{i}\left(i=\overline{1,3}\right.$ and $\left.\mathbf{a}_{i} \cdot \mathbf{b}_{j}=2 \pi \delta_{i, j}\right)$. We also assume that there are $M_{i} N_{i}$ primitive cells along the direction $i$, hence, $N=\prod_{i=1}^{3}\left[M_{i} N_{i}\right]$ is the total number of primitive cells in the crystal. The position of the primitive cells in the crystal is given by: $\mathbf{r}_{\mathbf{n}(i)}=$ $\sum_{k=1}^{3} n_{k}^{(i)} \mathbf{a}_{i}$, where $\mathbf{n}(i)$ labels the trio of integers $\left(n_{1}^{(i)}, n_{2}^{(i)}, n_{3}^{(i)}\right), i=\overline{1, N}$. We now consider the same crystal represented as an ensemble of supercells, each supercell comprising $N_{C}=N_{1} N_{2} N_{3}$ primitive cells and the bulk crystal consisting of $N_{S}=M_{1} M_{2} M_{3}$ supercells (with $N=N_{C} N_{S}$ ). The supercell direct lattice vectors are given by: $\mathbf{A}_{i}=N_{i} \mathbf{a}_{i}$, while the reciprocal supercell lattice vectors are given by: $\mathbf{B}_{i}=$ $1 / N_{i} \mathbf{b}_{i}$, with $i=\overline{1,3}$. There are $N_{C}$ supercell lattice reciprocal vectors falling within the primitive cell $\mathrm{BZ}$ :

$\mathbf{G}_{\mathbf{n}(j)}=\sum_{k=1}^{3} n_{k}^{(j)} \mathbf{B}_{i}=\sum_{k=1}^{3} \frac{n_{k}^{(j)}}{N_{k}} \mathbf{b}_{i}$,

where now $\mathbf{n}(j)$ is a trio of integers $\left(n_{1}^{(j)}, n_{2}^{(j)}, n_{3}^{(j)}\right)$, $j=\overline{1, N_{C}}$, running over the all the supercells in the bulk crystal. Any vector of the primitive-cell lattice $\mathbf{r}_{\mathbf{n}(i)}$ can be written in the supercell representation as $\mathbf{r}_{\mathbf{n}(i)}=\mathbf{R}_{s}+\boldsymbol{\rho}_{l}$, where $\mathbf{R}_{s}$ is the origin of the $s^{\text {th }}$ supercell, with $s$ labelling the supercells in the crystal, $s=\overline{1, N}_{S} ; \boldsymbol{\rho}_{l}$ is the position of the $l^{\text {th }}$ primitive cell in the $s^{\text {th }}$ supercell (relative to the supercell origin), and $l$ labels the primitive cells in the supercell, $l=\overline{1, N}_{C}$. On the other hand, any vector $\mathbf{k}$ in the first BZ of the primitive-cell lattice can be written in the supercell representation as: $\mathbf{k}=\mathbf{K}+\mathbf{G}_{\mathbf{n}(j)}$, where $\mathbf{K}$ is a vector in the first BZ of the supercell lattice and $\mathbf{G}_{\mathbf{n}(j)}$ is the corresponding translation vector from the reciprocal lattice of the supercell.

In order to unfold a band structure, it is important to understand how the BZ folds in the first place. The simplest way to visualise this is using a $2 \mathrm{D}$ square lattice example, shown in Fig. 3(A). The primitive cell BZ folds into the supercell BZ from all directions (see Fig. 3(B)), adding a level of complexity in generating the final band structure. Therefore, in order to unfold the supercell band structure, 'translation' vectors are used, which are simply the supercell reciprocal lattice vectors $\mathbf{G}_{n(j)}$ (defined in Eq.1), which fall within the first BZ of the primitive lattice. These translation vectors in the context of a $2 \mathrm{D}$ square lattice are shown in Fig. 3, which depicts the procedure used to unfold the folded supercell vectors employing the translation vectors labelled $\mathbf{G}_{1}=\mathbf{0}, \mathbf{G}_{8}$ and $\mathbf{G}_{6}$ (Fig. 3(C)-(E)). Fig. 3(F) shows how the folded vectors along a highsymmetry path in the supercell $\mathrm{BZ}$ can be unfolded to cover the corresponding path along a high-symmetry 


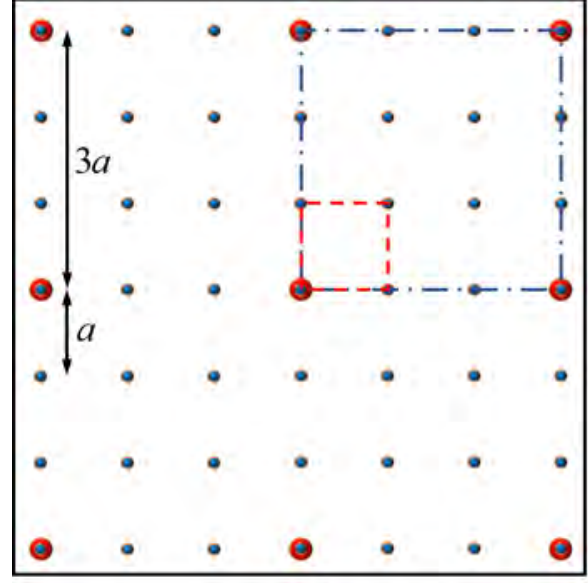

(A)

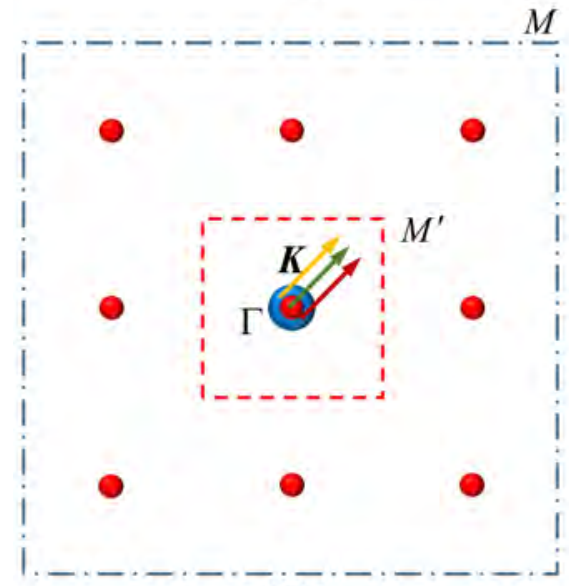

(C)

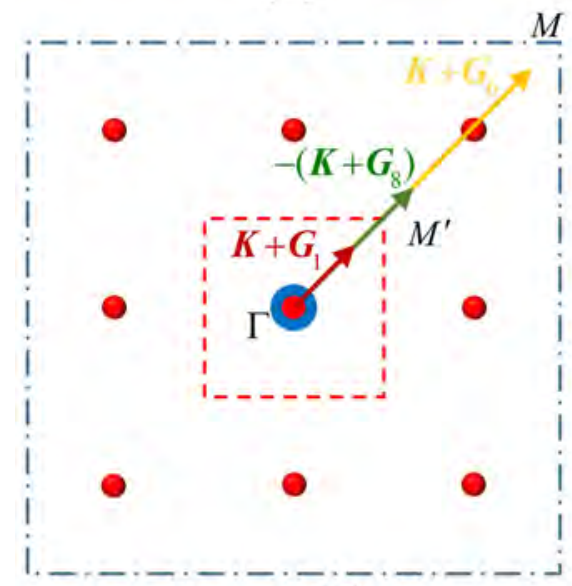

(E)

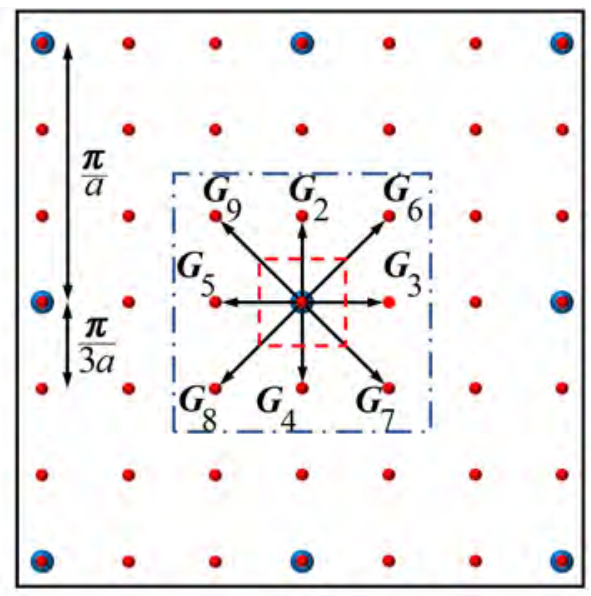

(B)

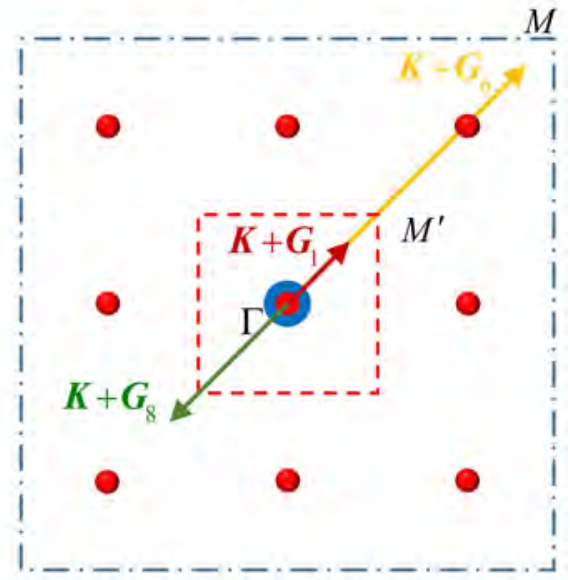

(D)

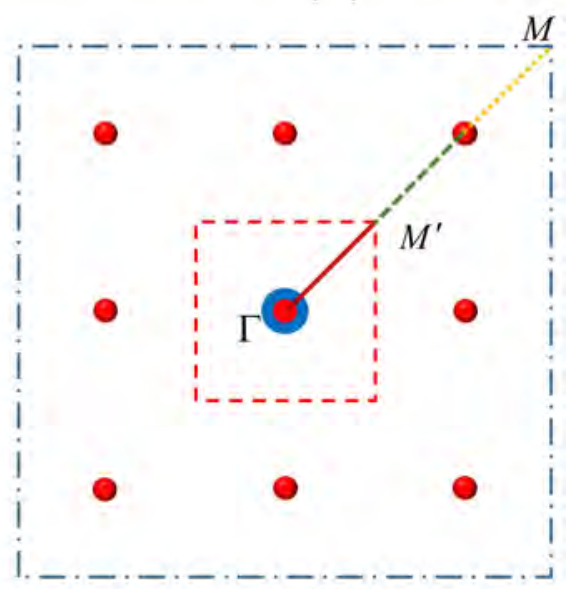

(F)

Figure 3. (Color online) Depiction of the unfolding process shown for a 2 D square lattice. Panel (A) shows a direct lattice (smaller dots) with a primitive cell of size $a$ (delineated by the dashed line) and a supercell lattice (larger dots; unit cell delineated by the dot-dashed line) comprising $N_{C}=9$ primitive cells. Panel (B) shows the reciprocal lattice for the primitive (larger dots) and supercell (smaller dots) configurations and the $\mathbf{G}_{\mathbf{n}(j)}$ vectors used in the text (for simplicity, the $\mathbf{G}_{1}=0$ is not shown). The first $\mathrm{BZ}$ of the primitive lattice is delineated by the dot dashed line and the first BZ of the supercell lattice is delineated by the dashed line. Panels (C)-(F) show the unfolding processes used to recreate a typical high-symmetry path. Panel (C) depicts three identical vectors, K, folded in the supercell BZ. Panel (D) shows the first step in the unfolding process, the translation of the folded vectors by the supercell reciprocal vectors, $\mathbf{G}_{0}, \mathbf{G}_{6}$ and $\mathbf{G}_{8}$, respectively. In panel panel (E), we exploit the degeneracy of the $\pm \mathbf{k}$ states to align the $\mathbf{k}=\mathbf{K}+\mathbf{G}_{8}$ vector along the $\Gamma$-M path. Panel $(\mathrm{F})$ shows that as the $\mathbf{K}$ folded vectors run along the $\Gamma$ - $\mathrm{M}^{\prime}$ path of the supercell $\mathrm{BZ}$, the resulting unfolded vectors cover the $\Gamma-\mathrm{M}$ path of the primitive-cell BZ. 
direction in the primitive-cell BZ (when $\mathbf{K}$ runs along the $\Gamma-\mathrm{M}^{\prime}$, the three unfolded vectors $\mathbf{k}$ cover the continuous, dashed and dot-dashed paths in Fig. 3(F), hence the whole $\Gamma$-M path in the BZ of the primitivecell lattice).

The reciprocal lattice vectors provide all possible positions in the BZ where the band structure can be unfolded, however, they do not define which energy levels need to be unfolded in which direction. Therefore, a relationship needs to be determined between the translation vectors and the eigenenergies for each wavevector in the band structure. This is achieved by expanding the supercell eigenstates as a superposition of the primitive-cell eigenstates. In the primitive-cell representation, the eigenstate of energy $E_{\eta}$ and wavevector $\mathbf{k}$ is:

$\left|\psi_{\eta}(\mathbf{k})\right\rangle=\sum_{i=1}^{N} \sum_{\mu=1}^{p} \sum_{\alpha} \frac{b_{\eta}^{(\alpha, \mu)}(\mathbf{k})}{\sqrt{N}} e^{i \mathbf{k} \cdot \mathbf{r}_{\mathbf{n}(i)}}\left|\alpha, \mu ; \mathbf{r}_{\mathbf{n}(i)}\right\rangle$

where $\mathbf{r}_{\mathbf{n}(i)}$ points to the origin of the $i^{\text {th }}$ primitive-cell in the crystal (with the summation index $i$ running over all primitive cells in the crystal, $i=\overline{1, N}) ; b_{p}^{(\alpha, \mu)}(\mathbf{k})$ are the expansion coefficients, and $\left|\alpha, \mu ; \mathbf{r}_{\mathbf{n}(i)}\right\rangle$ represents the atomic orbital $\alpha\left(s, p_{x}\right.$, etc. $)$ of the $\mu^{\text {th }}$ atom in the primitive cell (to accommodate polyatomic bases we assume $\mu=1, \ldots, p$ atoms per basis). Recalling that we can write $\mathbf{r}_{\mathbf{n}(i)}=\mathbf{R}_{s}+\boldsymbol{\rho}_{l}, \mathbf{k}=\mathbf{K}+\mathbf{G}_{\mathbf{n}(j)}$ and $N=N_{S} N_{C}$, the primitive-cell eigenstate becomes:

$$
\begin{array}{r}
\left|\psi_{\eta}\left(\mathbf{K}+\mathbf{G}_{\mathbf{n}(j)}\right)\right\rangle=\sum_{s=1}^{N_{S}} \sum_{l=1}^{N_{C}} \sum_{\mu=1}^{p} \sum_{\alpha} \frac{b_{\eta}^{(\alpha, \mu)}\left(\mathbf{K}+\mathbf{G}_{\mathbf{n}(j)}\right)}{\sqrt{N_{S} N_{C}}} \\
\times e^{i \mathbf{K} \cdot \mathbf{R}_{s}} e^{i\left(\mathbf{K}+\mathbf{G}_{\mathbf{n}(j)}\right) \cdot \boldsymbol{\rho}_{l}}\left|\alpha, \mu ; \mathbf{R}_{s}+\boldsymbol{\rho}_{l}\right\rangle,(3)
\end{array}
$$

where we have used $e^{i \mathbf{G}_{\mathbf{n}(j)} \cdot \mathbf{R}_{s}}=1$.

Similarly, the supercell eigenstate of energy $E_{\eta}$ and wavevector $\mathbf{K}$ is given by

$$
\begin{aligned}
\left|\Psi_{\eta}(\mathbf{K})\right\rangle= & \sum_{s=1}^{N_{S}} \sum_{l=1}^{N_{C}} \sum_{\mu=1}^{p} \sum_{\alpha} \frac{\beta_{\eta, l}^{(\alpha, \mu)}(\mathbf{K})}{\sqrt{N_{S}}} e^{i \mathbf{K} \cdot \mathbf{R}_{s}} \\
& \times\left|\alpha, \mu ; \mathbf{R}_{s}+\boldsymbol{\rho}_{l}\right\rangle,
\end{aligned}
$$

where the supercell "basis" contains now $p N_{C}$ atoms.

The supercell eigenstates can also be represented as superposition of the primitive cell eigenstates [17]:

$\left|\Psi_{\eta}(\mathbf{K})\right\rangle=\sum_{j=1}^{N_{C}} a_{\eta, \mathbf{n}(j)} \mid \psi_{\eta}\left(\mathbf{K}+\mathbf{G}_{\mathbf{n}(j)}\right\rangle$

where

$$
\begin{aligned}
& \mathcal{P}_{\eta, \mathbf{n}(j)}=\left|a_{\eta, \mathbf{n}(j)}\right|^{2} \\
& =\left\langle\psi_{\eta}\left(\mathbf{K}+\mathbf{G}_{\mathbf{n}(j)}\right) \mid \Psi_{\eta}(\mathbf{K})\right\rangle\left\langle\Psi_{\eta}(\mathbf{K}) \mid \psi_{\eta}\left(\mathbf{K}+\mathbf{G}_{\mathbf{n}(j)}\right)\right\rangle
\end{aligned}
$$

represents the probability that the eigenvalue $\eta$ should be unfolded with the translation vector $\mathbf{G}_{\mathbf{n}(j)}$. Using

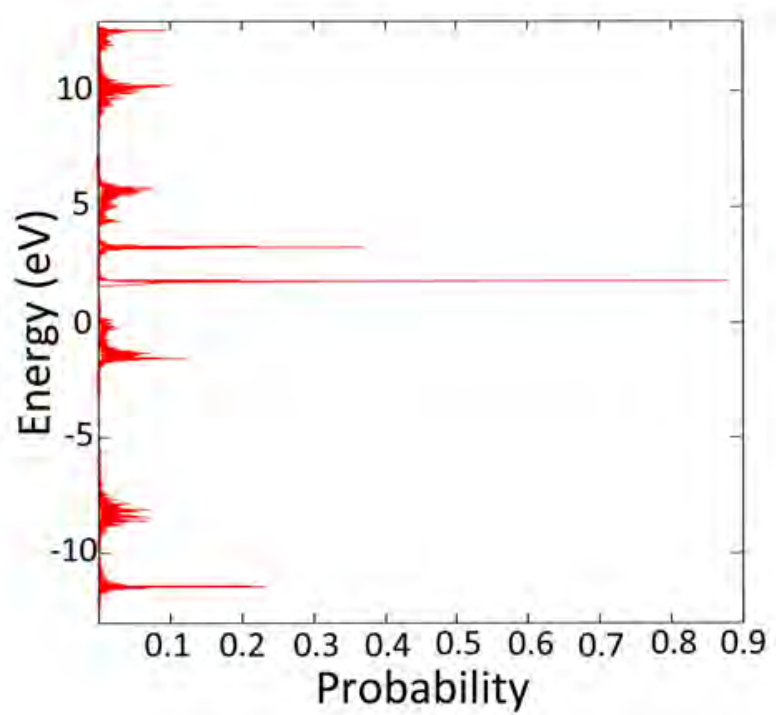

Figure 4. (Color online) Spectral function of a 1458 atom GaAsBi supercell with $10 \%$ bismuth fraction. Each peak represents at least one energy level in the effective band structure and is itself distributed across multiple energies resulting in a blurring effect when compared to the unfolded dispersion relation.

Eqs. 2-5, one can derive a linear system of $N_{C}$ equations for the probabilities $a_{\eta, \mathbf{n}(j)}[17]$ :

$$
\begin{aligned}
e^{-i \mathbf{K} \cdot \boldsymbol{\rho}_{l}} \beta_{\eta, l}^{(\alpha, \mu)} & (\mathbf{K})= \\
& \sum_{j=1}^{N_{C}} \frac{a_{\eta, \mathbf{n}_{j}}}{\sqrt{N_{C}}} b_{\eta}^{(\alpha, \mu)}\left(\mathbf{K}+\mathbf{G}_{\mathbf{n}(j)}\right) e^{i \mathbf{G}_{\mathbf{n}(j)} \cdot \boldsymbol{\rho}_{l}}
\end{aligned}
$$

Using the normalisation of the primitive-cell eigenstates, Eq. 7 can be solved for all eigenvalues $\eta$ and translation directions $\mathbf{n}(j)$ to yield:

$\mathcal{P}_{\eta, \mathbf{n}(j)}=\frac{1}{N_{C}} \sum_{\mu=1}^{p} \sum_{\alpha}\left|\sum_{l=1}^{N_{C}} e^{-i \mathbf{K} \cdot \boldsymbol{\rho}_{l}} \beta_{\eta, l}^{(\alpha, \mu)} e^{-i \mathbf{G}_{\mathbf{n}(j)} \cdot \boldsymbol{\rho}_{l}}\right|^{2}$

Equation (8) gives the probability, for each eigenvalue $\eta$ of an energy level and folded vector $\mathbf{K}$, to exist at a given unfolded wavevector $\mathbf{k}=\mathbf{K}+\mathbf{G}_{\mathbf{n}(j)}$. These spectral functions can then be analysed, defining which energies should be unfolded. In the case of a perfect supercell, the probabilities will be binary (i.e. 0 or 1) meaning that energies can be precisely associated to a translation vector and hence be precisely unfolded. However, for imperfect supercells, the spectral function becomes a series of distributed peaks, where multiple eigenvalues contribute towards a band in the unfolded band structure, as shown in Fig. 4. This is where the broadening of bands can be calculated. An example of this method is shown in Fig. 5, where a 6750 atom supercell of $\mathrm{GaAs}_{(1-x)} \mathrm{Bi}_{(x)}$ with a $10 \%$ bismuth fraction has been unfolded (it should be noted that only the peak of each band is plotted for clearer representation of the band structure). 


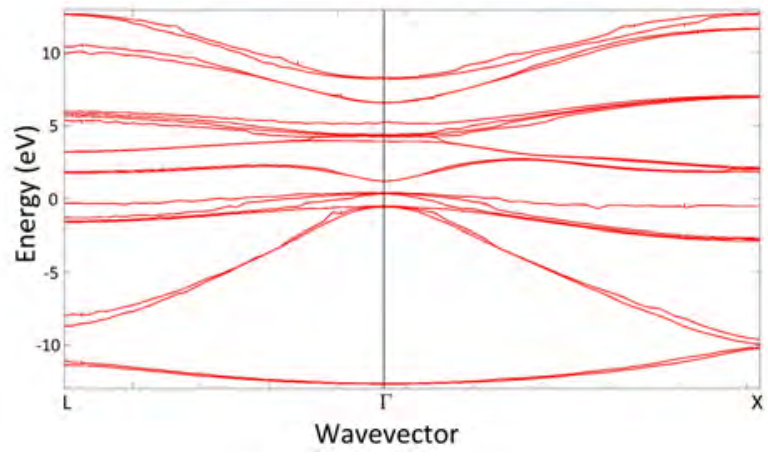

Figure 5. (Color online) Unfolded band structure of a 6750 atom GaAsBi supercell with $10 \%$ bismuth fraction. The mean of the peaks were chosen to depict the energy level for a clearer visual representation of the unfolded dispersion relation.

\section{Results}

The unfolding method presented above has been employed to unfold the band structures of $\mathrm{GaAs}_{(1-x)} \mathrm{Bi}_{(x)}$ for a number of bismuth fractions in order to investigate how the properties of this alloy develop. The main properties of interest, for changing bismuth concentration, are the band gap and the spin-orbit splitting energies because they define the expected wavelength at which the material will lase, and the condition for which $\mathrm{CHSH}$ Auger recombination should be suppressed. However, these unfolded band structures can also be used to investigate many other properties of the bismide alloy including gain calculations, Auger recombination rates for the bulk material and other material parameters including effective masses and band gap variation necessary for device simulations.

In order to calculate many of these material characteristics the band structure must also be known away from the high symmetry directions. Fig. 6 shows the band structure for a slice of the BZ along the $k_{z}=0$ plane for a GaAsBi alloy with $10 \%$ bismuth fraction. This displays the capability of the unfolding algorithm throughout the $\mathrm{BZ}$ demonstrating that the generated band structure can be safely used to investigate the properties of the GaAsBi alloy.

A key feature of bismuth alloys is the possibility to strongly manipulate the band positions due the size of the bismuth atom and its large spin-orbitsplitting energy. Therefore, it is necessary to simulate the levels, or more importantly the band gap and spin-orbit-splitting displacement as a function of the bismuth fraction. Fig. 7 shows the band gap and spin-orbit splitting energy as a function of bismuth fraction for 6750 atom GaAsBi supercells along with several experimental results[10]. The results predicted by our unfolding algorithm correlate strongly with the experimental results and other theoretical calculations[9]. More importantly, the results confirm a

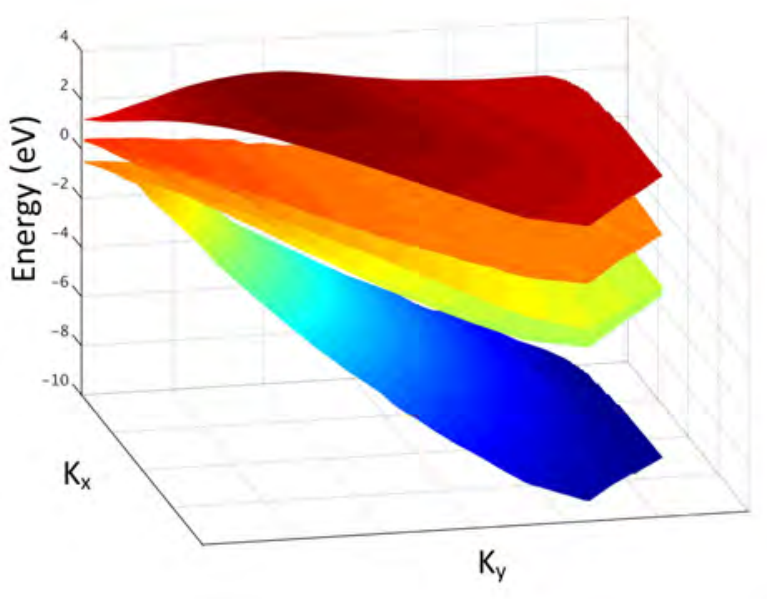

Figure 6. (Color online) A slice of the $\mathrm{BZ}$ along plane, $k_{z}=0$, of the unfolded band structure of a 6750 atom GaAsBi supercell with $10 \%$ bismuth fraction.

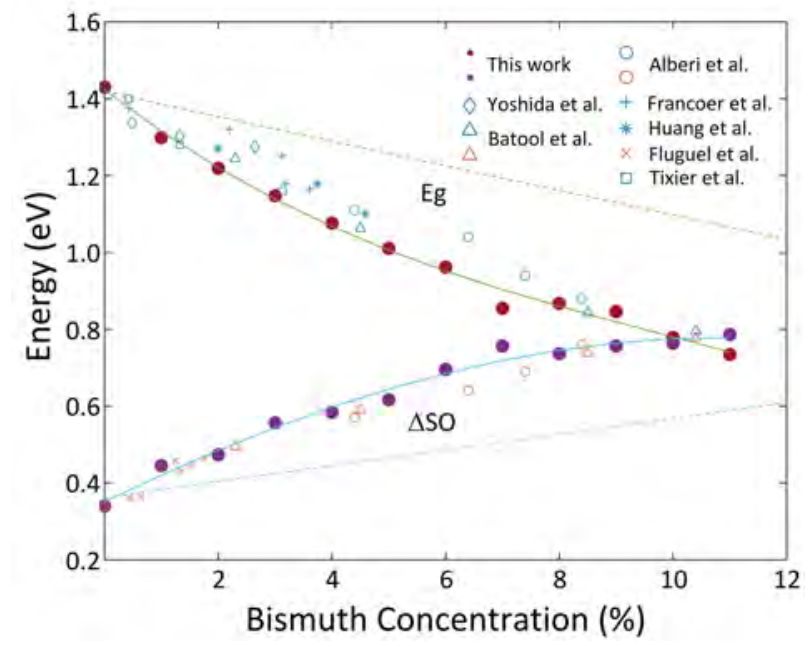

Figure 7. (Color online) Experimental and theoretical data on the dependence of the band gap and spin-orbit splitting energy as a function of the bismuth fraction. The simulation results correlate well with experiment data, and clearly identify the cross over region at $\sim 10 \%$ bismuth[10].

target for the growth of GaAsBi alloys of greater than $\sim 10 \%$ bismuth fraction, for which the SO band has been lowered such that the transition to the $\mathrm{HH}$ band is no longer possible and CHSH Auger recombination rates should be strongly suppressed.

The effective mass is also an important property to extract from the dispersion relation as is one of the common material parameters used for device simulation. However, the effective mass for a disordered crystal is more complex than that of a perfect crystal due to the loss of translational symmetry, which causes an effective distribution of effective masses. This can be further described by the knowledge of the broadening of the bands caused by 
defects in the crystal. Intrinsically, if the thickness of a band becomes finite, the curvature of that band becomes less well-defined.

Previous studies of GaAsN alloys, in which the nitrogen has a vastly different size and electronegativity to arsenic, have demonstrated a strong effect on the band structure[13]. Bismuth defects in GaAsBi are also vastly different and have a strong broadening effect[14] on the band structure, to the point that the effective mass becomes a poorly defined property. This effect is evident in Fig. 8, which depicts the band structure of the light-hole, heavy-hole and conduction bands near the $\Gamma$ point of the BZ for multiple configurations of a 2000 atom structure at $9 \%$ bismuth concentration. The first two (valence) bands are highly broadened to the point that the width of the band is greater than the displacement caused by its curvature making an effective mass fitting very unreliable. However, the conduction band appears far more stable indicating that it is much less effected by the introduction of bismuth, which agrees with the spectral function above where the conduction band has an overlap of nearly 1.0. A high overlap generally implies that the distribution of energies or the influence of the distribution is small. The opposite effect can also be found in the spectral functions for the valence bands, which have very low peaks and a large spread in the overlap. This broadening is mostly likely due to the presence of the bismuth defect band below the valence band edge[15]. The limited size of the supercell has a small contribution to the broadening but this can be easily overcome by increasing the size of the supercell or by averaging over many smaller supercell structures. It should be noted that this is only necessary due to the incredibly high accuracy required for measuring effective mass directly.

The electronic transport properties of GaAsBi alloys are still under debate with the trend of hole mobilities, as a function of bismuth content, varying quite drastically from experiment to experiment[23]. If the effective bands are highly broadened then the measured effective mass can therefore vary significantly only really converging when measured on a sufficiently large structure where the full range of mobility can be averaged out. However, as devices generally have fairly confined structures, this becomes less plausible, therefore, for device simulation, the variability will need to be accounted for.

Calculating the variability of effective masses can be achieved multiple ways. The simplest is to calculate the band structure of a large enough structure that it averages out all configurations. This would allow you to find the average effective mass and define an error on this value from the width of distributions in the spectral function. However, this becomes computationally infeasible for moderately sized supercells, so alternatively we can calculate the band structure of a larger number of smaller structures. This way, it would become eventually possible to build a density plot of bands where the peak indicates the average energy of that band and the width would provide the distribution of the energy values.

Using 135 different 2000-atom structures, the average effective masses (normalised by the values for GaAs) were calculated for bismuth concentrations varying from $0-12 \%$, as shown in Fig. 9. These values were calculated by measuring the curvature of the bands very close to the $\Gamma$ point.

The stability of the conduction band is reinforced by the trend of its averaged effective mass, which decreases gradually at approximately 0.006 per percentage of bismuth concentration. On the other hand, even the averaged effective mass of the valence bands are not completely stable especially in the case of the heavy-hole, but both display a general trend. The light-hole appears to rapidly increase up to $3 \%$ bismuth and then plateaus. The heavy-hole effective mass seems to mirror the light hole up to $6 \%$ but after that becomes fairly erratic, but maintaining a general decreasing trend. Further investigation is required to quantify the magnitude of the distribution behind these averaged effective masses.

There is a large difference between the effect of bismuth on the effective masses of the light-hole and the other two bands. This can be ascribed to positioning of the Bi defect band with respect to the respective bands. The Bi defect band intercepts the light-hole band close to the BZ centre, hence, strongly bowing the band. However, the heavy-hole band does not interact with the defect band until further away from the zone centre having a much smaller effect. The Bi defect's interaction with the conduction band can be considered negligible, and so the effective masses will only be perturbed by the Bi-induced strain effects in the lattice. Furthermore, a direct comparison of the results for carrier mobility from experiment to the results from this method is not straightforward due to the large variation in the experimental results. However, there is an apparent trend of decreased hole mobility (increased effective mass) that levels out at around $6 \%$ bismuth, which correlates well with the light-hole effective mass[23].

\section{Conclusion}

In summary, the supercell tight-binding approach to generating band structures for GaAsBi alloys correlates strongly with experimental results but is complex to analyse. However, using an unfolding algorithm, a dispersion relation can be generated that allows simple extraction of the alloys properties. Furthermore, the 
(a)

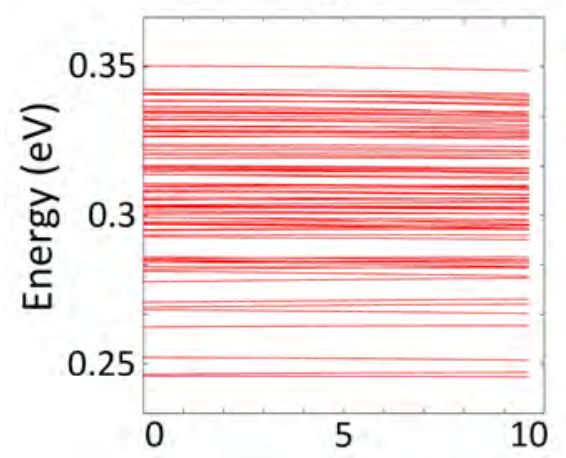

(b)

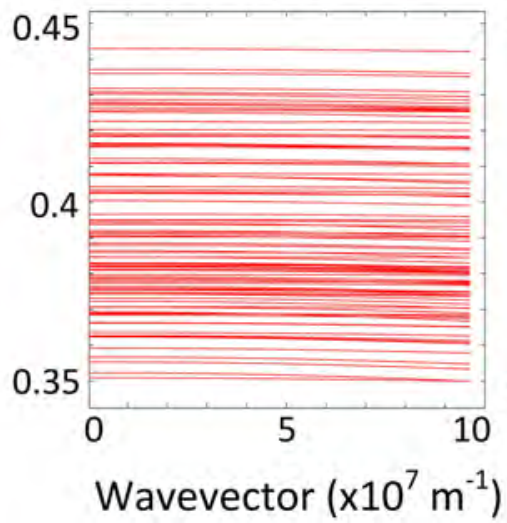

(c)

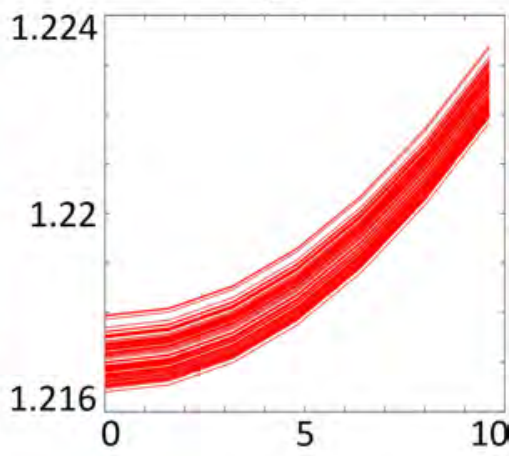

Figure 8. (Color online) Band structure plots of (a) light-hole, (b) heavy-hole and (c) conduction bands very close to the $\Gamma$ point in the BZ. Each plot contains the unfolded bands of 135 different 2000 atom structures that all have a bismuth concentration of $9 \%$. The first two plots show the broadening effect of the bismuth defect of the valence band, whereas the conduction band is far less effected.

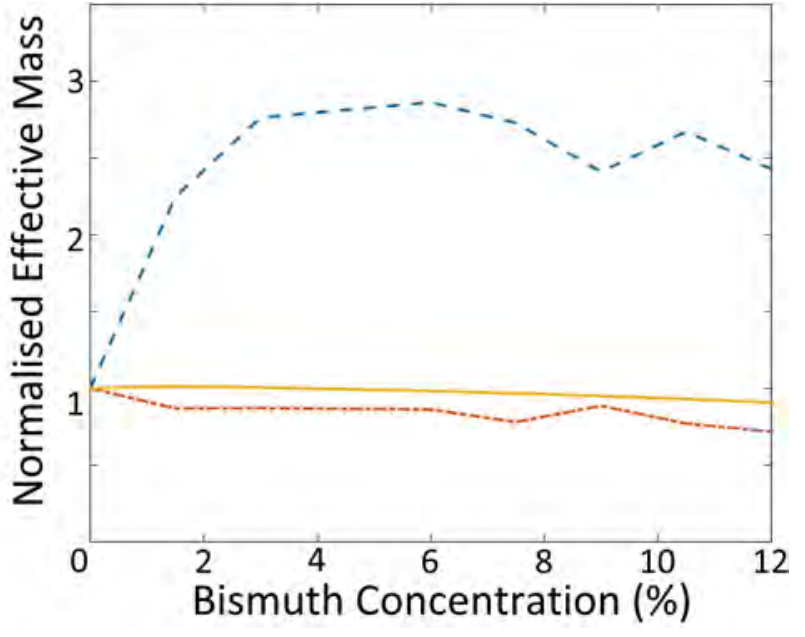

Figure 9. Effective masses as a function of bismuth concentration for the light-hole (dashed line), heavy-hole (dotdashed line) and conduction band (solid line). Both, heavy-hole and conduction bands, show a slight decrease in effective mass with increasing bismuth concentration, whereas the light-hole becomes significantly heavier.

unfolded band structure holds information about the effect of disorder and defects, which causes and blurring of the energy levels and hence will increase the variance on other properties like the effective mass.

The unfolding of GaAsBi has shown that simplistic approaches to calculating the effective masses may no longer be an accurate enough estimation as the broadening of bands causes transport properties to become highly dependant on local structural configurations. Therefore, simulation of devices will need to account for this variability.

\section{Acknowledgements}

We acknowledge EPSRC funding support under grants $\mathrm{EP} / \mathrm{H} 005587 / 1, \mathrm{EP} / \mathrm{L} 02263 \mathrm{X} / 1$ (EP/M008576/1) and EP/M027791/1. The authors confirm that data underlying the findings are available without restriction. Details of the data and how to request access are available from the University of Surrey publications repository: http://epubs.surrey.ac.uk/809461/.

\section{References}

[1] S. J. Sweeney and A. F. Phillips, Photonics Technology Letters 10(8), 1076 (1998).

[2] S. J. Sweeney, A. R. Adams, M. Silver, E. P. O'Reilly, J.R. Watling, A. B. Walker, and P. J. A. Thijs, Phys. Stat. Sol. B 211, 525 (1999).

[3] K. OBrien, S. J. Sweeney, A. R. Adams, B. N. Murdin, A. Salhi, Y. Rouillard, and A. Joullie, Appl. Phys. Lett. 89, 051104 (2006).

[4] S. J. Sweeney, Z. Batool, K. Hild, S. R. Jin, and T. J. C. Hosea, ICTON 2011

[5] S. J. Sweeney, Patent WO 2010/149978 (2010)

[6] K. Alberi, O. D. Dubon, W. Walukiewicz, K. M. Yu, K. Bertulis, and A. Krotkus, Appl. Phys. Lett. 91, 051909 (2007).

[7] S. Francoeur, M.-J. Seong, A. Mascarenhas, S. Tixier, M. Adamcyk, and T. Tiedje, Appl. Phys. Lett. 82, 3874 (2003).

[8] S. Tixier, M. Adamcyk, T. Tiedje, S. Francoeur, A. Mascarenhas, Peng Wei, and F. Schiettekatte, Appl. Phys. Lett. 14, 2245 (2003).

[9] M. Usman, C. Broderick, A. Lindsay, and E. O'Reilly, Phys. Rev. B 84, 1 (2003).

[10] Z. Batool, K. Hild, T. J. C. Hosea, X. Lu, T. Tiedje, and S. J. Sweeney, J. Appl. Phys. 111, 113108 (2012).

[11] K. Alberi, J. Wu, W. Walukiewicz, K. Yu, O. Dubon, S. Watkins, C. Wang, X. Liu, Y. J. Cho, and J. Furdyna, Phys. Rev. B 75, 045203 (2007).

[12] H. X. Deng, J. Li, S. S. Li, H. Peng, J. B. Xia, L. W. Wang, and S-H. Wei, Phys. Rev. B 82, 193204 (2010).

[13] S-H. Wei and A Zunger, Phys. Rev. Letters 76, 664 (1996) 
[14] O. Rubel, A. Bokhanchuk, S. Ahmed, and E. Assmann, Phys. Rev. B 90, 115202 (2014)

[15] C. A. Broderick, M. Usman, and E. P. O'Reilly, Phys. Stat. Sol. B 250, 773 (2013).

[16] Y. Zhang, A. Mascarenhas, and L.-W. Wang, Phys. Rev. B 71, 155201 (2005).

[17] T. Boykin, and G. Klimeck, Phys. Rev. B 71, 115215 (2005).

[18] V. Popescu, and A. Zunger, Phys. Rev. B 85, 085201 (2012).

[19] W. Ku, T. Berlijn, and C. C. Lee, Phys. Rev. Lett. 104, $216401(2010)$.

[20] O. L. Lazarenkova1, P. von Allmen, F. Oyafuso, S. Lee, and G. Klimeck, Appl. Phys. Lett. 85, 4193 (2004).

[21] S. Steiger, and M. Povolotskyi, IEEE Transactions on Nanotechnology 10, 1464 (2011).

[22] J. Poulson, B. Marker, R. van de Geijn, J. Hammond, and N. Romero, ACM Transactions on Mathematical Software 39(2), 13:1 (2013)

[23] G. Pettinari, A. Polimeni, M. Capizzi, H. Engelkamp, P. C. M. Christianen, J. C. Maan, A. Patané, and T. Tiedje, Phys. Stat. Sol. B 250, 779 (2013) 